to be any abnormal laxity of the joints generally. The limb was kept on a splint and evaporating lotions applied, and in a fortnight's time he was able to do light work, the wrist being still somewhat weak.

Remarks.-The mechanism of dislocation seems to have been as follows:- When the boy stumbled, his grasp of the moving cask tightened, and in endeavouring to recover himself the whole weight of his body was exerted on his right arm, so putting the ligaments of the wrist joint on the stretch. Almost immediately the morement of the arm backwards was arrested by the elbow sharply striking the other cask behind. The hand being fixed and bent backwards, the radius and ulna were thus easily jerked out of place. It was remarkable how easily the different bony parts involved could be recognised almost as if one were examining the bare skeleton. Moreover, the dislocation was quite uncomplicated, for the wrist was frequently and carefully examined by myself and others, but no thickening of bone or other sign of fracture in any part could at any time $b \rightarrow$ detected. The peculiar and unusual deformity, the straightness of the hand, the relative position of the styloid processes, which were entire, the arch of the carpal bones, the absence of crepitus, and the ability to supinate fully after complete pronation, were sufficient aids to diagnosis from other injuries at the wrist joint. Leytonstone.

\section{COCAINE EXTERNALLY AND INTERNALLY.}

\section{By G. Walter Steeves, M.D., \&c.}

The value of cocaine as a local anæsthetic is now well established, and few who have carefully tested its use, either by the simple application of a solution to the skin or mucous membrane or by hypodermic injection, will be sceptical as to its efficacy. During the past two years I have used it pretty largely in general practice, and the interest attaching to the extent and variety of its applicability, both in medicine and surgery, induces me to add this note.

Externally I have found cocaine useful in neuralgias, two obstinate cases of sciatica yielding under its application ; in operating on cysts and small tumours, such as ordinary sebaceous and Meibomian cysts; a fibro-cellular tumour, about two inches in diameter, was removed from the gluteal region of an old lady without the slightest pain; in pruritus ani; in stitching two cases of ruptured perineum, where the laceration was considerable; in opening abscesses in various parts; in coccydynia; in two cases of chronic articular rheumatism, where the excessive nocturnal pain resisted ordinary treatment. As an ointment I am finding its application of great assistance in the treatment of an obstinate case of vaginismus.

Internally I believe the drug to have special efficacy in certain cases of gastralgia. In two instances, one of which was accompanied by intense gastric irritability (all food and liquid being immediately vomited), the exhibition of the drug was followed by almost immediate relief. Other treatment by morphia, belladonna, \&c., had been previously tried, with no beneficial results. One of the above cases was that of a hysterical young lady under my care about a year ago, who, in addition to excessive gastric pain and epigastric tenderness, suffered from a general condition of hyperæasthesia. A notice of this patient is particularly interesting, as she had been under my observation for ten days with no improvement whatever, but after the cocaine had been administered for twenty-four hours the pain was subdued, and remained so. She has not had an attack since, although previously to this similar seizures had been periodical, especially on the approach of menstruation.

The above heterogeneous collection at least illustrates some of the uses to which cocaine may be applied by the general practitioner, and possibly may suggest others. In a recent issue of THE LANCET (August 6th) there is mentioned the case of a young woman lately under Dr. Frey of Bêkéx, in which a general condition of hyperæsthesia was associated with intense irritability of stomach and constant vomiting, where cocaine promptly relieved all alarming symptoms. We may fairly hope that as such cases of severe functional and "sympathetic" stomach derangement are so benefited, some of the distressing symptoms which so frequently accompany internal organic disease may also be alleviated by its use; so far, however, I have seen no mention of its exhibition in the latter class of cases. Its antiseptic influence might also be in its favour.
Internally I have prescribed the hydrochlorate (dissolved in water) in quarter-grain doses every four hours, or oftener when the gastric pain was excessive. For external application the solutions have varied from 4 to 10 per cent, in strength. Liverpool.

\section{TWO MIDWIFERY CASES.} BY J. H. BATTYE, M.D.

DURING the week ending August 20th I met with two remarkable cases in midwifery wbich I desire to place on record, as both are to me unique. One has a bearing on Dr. Oliver's "Notes on Sterility," which recently appeared in THE LANCET, and the other is remarkable for its prolonged first stage.

On August 18th, 1887, after twenty-four years and five months of married life with her present and only husband, at the age of forty-five I delivered a tradesman's wife of her first-born child, a male, at full term, the labour being perfectly natural and lasting twenty-eight hours from the first pains till the expulsion of the placenta. The patient is rather stout and has enjoyed very good health, and she did not suffer during gestation. There was not the usual morning sickness or any constitutional disturbance to show pregnancy; and when I first saw her on May 23rd she came to me fearing an unnatural growth, and 1 did not discover that she was pregnant, as in consequence of the thickness of the abdominal walls I could not feel the uterus properly; but when I next saw her, on July 13th, a great deal of the adipose tissue had disappeared, and I had no difficulty in making out the uterus and feeling the movements of the child. Before labour came on I must admit I felt somewhat anxious about her on account of her age, and because it was to me a unique case; howerer, my anxiety proved quite needless, as she is able to suckle her child, which is strong and vigorous, and both up to the present time have progressed as well as anyone could wish.

As a contrast to the above case with regard to duration of labour in primiparæ, another case, occurring the same week and completed the same day, gave me a record of the longest first stage of labour 1 have yet met with. This occurred in a coachman's wife, aged thirty, to whom I was called at 11 P.M. on Tuesday, Aug. 16th, pains having commenced at 10 A.M. On examination the os uteri would admit only the tip of the finger, so I gave her a dose of opium (incture of opium, $15 \mathrm{~min}$.) to enable her to get some sleep, and left her. In spite of the dose of opium, she had little sleep because of the pains; and on calling at 9 A.M. on Aug. 17th and examining, although the pains were good, I found the os very little dilated compared with the previous evening, not the size of a threepenny piece. At 4.30 P.M., being in the neighbourhood, I called again and found the os dilated to the size of a sixpence, not rigid, but soft, and the pains good, every ten minutes. At 11 P.M. I was called away to the case previously related, which occurred some five miles distant from this; and on my return on Thursday afternoon at 5.20 P.M., I found the os only the size of a shilling, although up till noon on the 18th (Thursday) the pains had continued every ten minutes. After noon till an hour before my return they had not been so frequent, but they occurred then at intervals of every five minutes while I was present, and yet there appeared no rigidity of the os. Having been in labour so long, I determined upon fetrhing Barnes's dilating bags, but before doing so I pushed up the head a little, letting down some liquor amnii, and now for the first time I felt the bulging of the membranes. On my return with Barnes's bags at 6 P.M. I found the os dilated beyond the largest size, and at $7.30 \mathrm{P}$ M. I ruptured the membranes, as the os was completely dilated. I had, however, to complete delivery by forceps at 9 P.M. in consequence of the exhaustion of the patient.

My explanation of this prolonged first stage of labour is this:- The patient had had a remarkably sick gestation, having vomited during labour till 9 A.Mr. on the 18th; that by the act of vomiting the child, which presented in the first cranial position, had been driven down so low in the pelvis that the liquor amnii could not get past the head until I pushed it back at 5.20 P.M. on the $18 \mathrm{th}$, when for the first time I could make sure that the membranes were entire. According to the times I have given, it will be seen that the duration of the first stage was fifty-seven hours and a half. The patient has since made uninterrupted progress towards recovery.

Denbigh-street, S.W. 